\title{
Cosmological Constraints on Lorentz Violation in Electrodynamics
}

\author{
V. Alan Kostelecký and Matthew Mewes \\ Physics Department, Indiana University, Bloomington, Indiana 47405
}

(Received 23 July 2001; published 29 November 2001)

\begin{abstract}
Infrared, optical, and ultraviolet spectropolarimetry of cosmological sources is used to constrain the pure electromagnetic sector of a general Lorentz-violating standard-model extension. The coefficients for Lorentz violation are bounded to less than $3 \times 10^{-32}$.
\end{abstract}

DOI: 10.1103/PhysRevLett.87.251304

Lorentz violation is a promising candidate signal for Planck-scale physics [1]. For instance, it could arise in string theory [2] and is a basic feature of noncommutative field theories [3]. In quantum field theory at attainable energies, small violations can be incorporated into the standard model to yield a general Lorentz-violating standard-model extension [4]. Its Lagrangian consists of all possible observer Lorentz scalars formed from standardmodel fields while allowing for coupling coefficients with Lorentz indices. All renormalizable and gauge invariant terms relevant at low energies are explicitly known.

The standard-model extension predicts definite experimental signals. In the fermion sector of the theory, various experiments have bounded coefficients for Lorentz violation. However, relatively little is known experimentally about the implications of the standard-model extension for the properties of light. In particular, no bounds have been placed on the $C P T$-even coefficients for Lorentz violation in the photon sector. In this work, we study these terms and use spectropolarimetry of cosmological sources to obtain stringent bounds on Lorentz violation comparable to the best current limits in the fermion sector.

Extracted from the standard-model extension, the Lorentz-violating electrodynamics can be written in terms of the usual field strength $F_{\mu \nu} \equiv \partial_{\mu} A_{\nu}-\partial_{\nu} A_{\mu}$, which incorporates the electric field $\vec{E}$ and the magnetic field $\vec{B}$. The relevant Lagrangian terms are [4,5]

$$
\mathcal{L}=-\frac{1}{4} F_{\mu \nu} F^{\mu \nu}-\frac{1}{4}\left(k_{F}\right)_{\kappa \lambda \mu \nu} F^{\kappa \lambda} F^{\mu \nu} .
$$

The second term is $C P T$ even and Lorentz violating. Its coefficient $\left(k_{F}\right)_{\kappa \lambda \mu \nu}$ is dimensionless. It has the symmetries of the Riemann tensor and zero double trace, so it contains 19 independent real components.

The modified inhomogeneous Maxwell equations obtained from Eq. (1) are

$$
\partial_{\alpha} F_{\mu}{ }^{\alpha}+\left(k_{F}\right)_{\mu \alpha \beta \gamma} \partial^{\alpha} F^{\beta \gamma}=0 .
$$

These are supplemented with the usual homogeneous Maxwell equations $\partial_{\mu} \widetilde{F}^{\mu \nu}=0$. For a plane electromagnetic wave with wave 4-vector $p^{\alpha}=\left(p^{0}, \vec{p}\right)$, we have $F_{\mu \nu}(x)=F_{\mu \nu}(p) e^{-i p_{\alpha} x^{\alpha}}$. The homogeneous equations ensure the absence of single magnetic poles, $\vec{p} \cdot \vec{B}=0$, and provide the usual Faraday law, $p^{0} \vec{B}=\vec{p} \times \vec{E}$. This
PACS numbers: 98.80.Es, 11.30.Cp, 12.60.-i, 41.20.Jb

can be used to eliminate $\vec{B}$ in the modified inhomogeneous equations, yielding the modified Ampère law [4]

$$
M^{j k} E^{k} \equiv\left(-\delta^{j k} p^{2}-p^{j} p^{k}-2\left(k_{F}\right)^{j \beta \gamma k} p_{\beta} p_{\gamma}\right) E^{k}=0 .
$$

The matrix $M^{j k}$ is real and symmetric, which can be shown to imply lossless propagation. The modified Coulomb law $p^{j} M^{j k} E^{k}=0$ follows from this equation, in parallel with the usual case.

For nontrivial solutions to Ampère's law, we must require $\operatorname{det} M^{j k}=0$. This condition provides the dispersion relation between $p^{0}$ and $\vec{p}$. Some calculation shows that, to leading order in the coefficients $\left(k_{F}\right)_{\kappa \lambda \mu \nu}$, the solutions to the dispersion relation take the form

$$
p_{ \pm}^{0}=(1+\rho \pm \sigma)|\vec{p}|,
$$

where $\rho=-\frac{1}{2} \tilde{k}_{\alpha}{ }^{\alpha}$ and $\sigma^{2}=\frac{1}{2}\left(\tilde{k}_{\alpha \beta}\right)^{2}-\rho^{2}$, with $\tilde{k}^{\alpha \beta} \equiv$ $\left(k_{F}\right)^{\alpha \mu \beta \nu} \hat{p}_{\mu} \hat{p}_{\nu}$ and $\hat{p}^{\mu} \equiv p^{\mu} /|\vec{p}|$. The solutions (4) describe behavior similar to that of spatial dispersion in an optically inactive but anisotropic medium. In particular, the vacuum is birefringent: light typically propagates as two different independent modes. Substitution of the two solutions (4) into the Ampère law (3) determines the electric fields $\vec{E}_{ \pm}$and hence the polarization of the two modes. For each mode, the group velocity $\overrightarrow{\boldsymbol{v}}_{g \pm} \equiv \vec{\nabla}_{\vec{p}} p_{ \pm}^{0}$ typically differs from the phase velocity $\vec{v}_{p_{ \pm}} \equiv p_{ \pm}^{0} \vec{p} / \vec{p}^{2}$.

At leading order in $\left(k_{F}\right)_{\kappa \lambda \mu \nu}$, the fields $\vec{E}_{ \pm}$are orthogonal and each is also perpendicular to $\vec{v}_{g \pm}$. The orthogonality implies that $\vec{E}_{ \pm}$span the set of all possible polarizations, and so the unit vectors $\hat{\varepsilon}_{ \pm} \equiv \vec{E}_{ \pm} /\left|\vec{E}_{ \pm}\right|$ form a basis for polarization at this order. The electric field can then be decomposed as $\vec{E}(x)=\left(E_{+} \hat{\varepsilon}_{+} e^{-i p_{+}^{0} t}+\right.$ $\left.E_{-} \hat{\varepsilon}_{-} e^{-i p_{-}^{0} t}\right) e^{i \vec{p} \cdot \vec{x}}$. Since the phase velocities of the two modes differ, their relative phase changes as a wave propagates through free space. The resulting change in the polarization state of the wave is determined by the relative phase change

$$
\Delta \phi=\left(p_{+}^{0}-p_{-}^{0}\right) t \approx 2 \pi \Delta v_{p} L / \lambda \approx 4 \pi \sigma L / \lambda,
$$

where $\Delta v_{p}$ is the difference in phase velocities, $\lambda$ is the wavelength, and $L$ is the distance the radiation has traveled. The distance dependence implies that, for sources at very large distances, tiny differences in the phase velocities may be observable. 
In recent years, high-quality spectropolarimetry of distant galaxies at infrared, optical, and ultraviolet frequencies has been performed [6-15]. These observations correspond to $L / \lambda$ greater than $10^{31}$. We can therefore anticipate that measurements of polarization parameters of order 1 should provide sensitivity of order $10^{-31}$ or better to components of $\left(k_{F}\right)_{\kappa \lambda \mu \nu}$. The inverse dependence of $\Delta \phi$ on wavelength is a special feature exploited here to obtain a definite bound on $\left(k_{F}\right)_{\kappa \lambda \mu \nu}$.

In spectropolarimetry, the quantitative measurement of polarization is usually described via Stokes parameters, defined in a frame where the 3-axis coincides with the direction of propagation [16]. Introducing unit vectors $\hat{e}_{1}$ and $\hat{e}_{2}$ along the 1- and 2-axes and the corresponding electric field components as $E_{1}$ and $E_{2}$, the Stokes parameters can be taken as

$$
\begin{aligned}
\left(s^{0}, \vec{s}\right) \equiv & \left(\left|E_{1}\right|^{2}+\left|E_{2}\right|^{2},\left|E_{1}\right|^{2}-\left|E_{2}\right|^{2},\right. \\
& \left.2 \operatorname{Re} E_{1}^{*} E_{2}, 2 \operatorname{Im} E_{1}^{*} E_{2}\right) \\
= & s^{0}(1, \cos 2 \chi \cos 2 \psi, \cos 2 \chi \sin 2 \psi, \sin 2 \chi),
\end{aligned}
$$

where $\chi$ and $\psi$ are the usual polarization angles. For convenience, we normalize throughout to $s^{0}=1$. In the present context, nonzero coefficients $\left(k_{F}\right)_{\kappa \lambda \mu \nu}$ leading to a nonzero $\sigma$ imply a finite phase shift $\Delta \phi$ in the radiation from a cosmological source, which in turn affects the Stokes vector $\vec{s}(\psi, \chi)$ determining its polarization. To set a bound on Lorentz violation, we must therefore first establish quantitatively the relationships between $\left(k_{F}\right)_{\kappa \lambda \mu \nu}$, $\sigma, \Delta \phi$, and $\vec{s}(\psi, \chi)$.

We begin our analysis by expressing $\sigma$ directly using the 19 independent components of $\left(k_{F}\right)_{\kappa \lambda \mu \nu}$. Since $\sigma^{2}$ is a quadratic form in $\left(k_{F}\right)_{\kappa \lambda \mu \nu}$, we can choose 19 independent components $k_{A}, A, B=1, \ldots, 19$, of $\left(k_{F}\right)_{\kappa \lambda \mu \nu}$ and can write $\sigma^{2}=S_{A B} k_{A} k_{B}$. The $19 \times 19$ matrix $S_{A B}$ is symmetric and depends on the direction of propagation. Some calculation shows that there exists a directionindependent similarity transformation such that $S_{A B}$ takes the form of a $19 \times 19$ matrix with only a $10 \times 10$ nonzero block. Therefore, $S_{A B}$ has rank 10, and only ten linearly independent combinations $k^{a}, a=1, \ldots, 10$, of $\left(k_{F}\right)_{\kappa \lambda \mu \nu}$ appear in $\sigma$. An acceptable choice for these ten combinations is

$$
\begin{aligned}
k^{a}= & \left(\left(k_{F}\right)^{0213},\left(k_{F}\right)^{0123},\left(k_{F}\right)^{0202}-\left(k_{F}\right)^{1313},\left(k_{F}\right)^{0303}-\left(k_{F}\right)^{1212},\left(k_{F}\right)^{0102}+\left(k_{F}\right)^{1323},\left(k_{F}\right)^{0103}-\left(k_{F}\right)^{1223},\right. \\
& \left.\left(k_{F}\right)^{0203}+\left(k_{F}\right)^{1213},\left(k_{F}\right)^{0112}+\left(k_{F}\right)^{0323},\left(k_{F}\right)^{0113}-\left(k_{F}\right)^{0223},\left(k_{F}\right)^{0212}-\left(k_{F}\right)^{0313}\right) .
\end{aligned}
$$

It now follows that $\sigma^{2}=\tilde{S}_{a b} k^{a} k^{b}$, where $\tilde{S}_{a b}$ is symmetric and direction dependent. The other nine linearly independent combinations of $\left(k_{F}\right)_{\kappa \lambda \mu \nu}$ play no role in birefringence, and bounding them will be the subject of a separate investigation. For definiteness, the reference inertial frame in which the $k^{a}$ are specified by Eq. (7) is chosen to be compatible with celestial equatorial coordinates, with the 3 -axis aligned along the celestial north pole at equinox 2000.0 at a declination of $90^{\circ}$. The $1-$ and 2-axes are at a declination of $0^{\circ}$ and a right ascension of $0^{\circ}$ and $90^{\circ}$, respectively. The goal is to bound the ten quantities $k^{a}$ defined in this frame.

The form of $\tilde{S}_{a b}$ is cumbersome and is omitted here. A more convenient expression for $\sigma^{2}$ can be obtained by calculating within a special inertial frame. The idea is to use observer rotation invariance to choose a "primed" frame in which $\hat{p}^{\mu}$ has leading-order form $\hat{p}^{\prime \mu}=(1 ; 0,0,1)$, to solve for the Lorentz scalar $\sigma^{2}$ in this frame in terms of $\left(k_{F}\right)_{\kappa \lambda \mu \nu}^{\prime}$, and then to use the rotation between the celestial equatorial frame and the primed frame to express $\left(k_{F}\right)_{\kappa \lambda \mu \nu}^{\prime}$ in terms of $\left(k_{F}\right)_{\kappa \lambda \mu \nu}$. To match standard polarimetric conventions, we choose the primed-frame basis vector $\hat{e}_{3}^{\prime}$ to point from the source towards the Earth, while $\hat{e}_{1}^{\prime}$ points south [17].

Solving the Ampère law (3) in the primed frame gives $\rho=\frac{1}{2}\left(\tilde{k}^{\prime 11}+\tilde{k}^{\prime 22}\right)$ and $\sigma^{2}=\left(\tilde{k}^{\prime 12}\right)^{2}+\frac{1}{4}\left(\tilde{k}^{\prime 11}-\tilde{k}^{\prime 22}\right)^{2}$. This form for $\sigma^{2}$ suggests defining an angle $\xi$ such that $\tilde{k}^{\prime 12}=\sigma \sin \xi$ and $\frac{1}{2}\left(\tilde{k}^{\prime 11}-\tilde{k}^{\prime 22}\right)=\sigma \cos \xi$. The angle $\xi$ determines the minimal linear combinations of $\left(k_{F}\right)_{\kappa \lambda \mu \nu}$ relevant for polarimetry of a specific source. Note that $\xi$ is frame dependent, unlike $\rho$ and $\sigma$.

At leading order, the polarization basis vectors in this frame obey $\hat{\varepsilon}_{ \pm} \propto(\sin \xi, \pm 1-\cos \xi, 0)$ and are linearly polarized. The corresponding Stokes vectors are $\vec{s}_{ \pm}=$ $\pm(\cos \xi, \sin \xi, 0)$. The propagation from the source to the Earth generates a relative phase change $\Delta \phi$ specified by Eq. (5). The corresponding effect on the Stokes vector $\vec{s}(\psi, \chi)$ for the radiation can be regarded as a rotation by $\Delta \phi$ about the basis vector $\vec{s}_{+}$. This typically changes both $\psi$ and $\chi$.

The change in polarization depends not only on the coefficients $\left(k_{F}\right)_{\kappa \lambda \mu \nu}$, the wavelength $\lambda$, and the distance to the source $L$, but also on the initial polarization. For cosmological sources, there is usually no way to determine independently the polarization produced at the source. We adopt instead the reasonable assumption that the source polarization is constant over the relatively narrow band of wavelengths being considered for each source. The quantity of interest is then the change in relative phase $\delta \phi=4 \pi \sigma\left(L / \lambda-L / \lambda_{0}\right)$ between a wavelength $\lambda$ and a reference wavelength $\lambda_{0}$.

The rotation of the Stokes vector can be expressed via a Mueller matrix $m^{j k}$, often used to describe the effects of filters and polarizers on light [16]. The change in the Stokes vector is given by $s^{j}(\psi, \chi)=m^{j k}(\delta \phi) s^{k}\left(\psi_{0}, \chi_{0}\right)$, where $\psi_{0}, \chi_{0}$ are reference polarization angles. Some algebra reveals that 


$$
m^{j k}(\delta \phi)=\left(\begin{array}{ccc}
\cos ^{2} \xi+\sin ^{2} \xi \cos \delta \phi & \sin \xi \cos \xi(1-\cos \delta \phi) & \sin \xi \sin \delta \phi \\
\sin \xi \cos \xi(1-\cos \delta \phi) & \sin ^{2} \xi+\cos ^{2} \xi \cos \delta \phi & -\cos \xi \sin \delta \phi \\
-\sin \xi \sin \delta \phi & \cos \xi \sin \delta \phi & \cos \delta \phi
\end{array}\right)
$$

The polarization angle $\chi$, which measures the amount of circular polarization, is unavailable in most published literature on astronomical spectropolarimetry. We therefore focus attention here on the change $\delta \psi=\psi-\psi_{0}$ in the polarization angle $\psi$. After some algebra, we find

$$
\delta \psi=\frac{1}{2} \tan ^{-1} \frac{\sin \tilde{\xi} \cos \zeta_{0}+\cos \tilde{\xi} \sin \zeta_{0} \cos \left(\delta \phi-\phi_{0}\right)}{\cos \tilde{\xi} \cos \zeta_{0}-\sin \tilde{\xi} \sin \zeta_{0} \cos \left(\delta \phi-\phi_{0}\right)},
$$

where $\tilde{\xi}=\xi-2 \psi_{0}$ and $\phi_{0} \equiv \tan ^{-1}\left(\tan 2 \chi_{0} / \sin \tilde{\xi}\right)$, $\zeta_{0} \equiv \cos ^{-1}\left(\cos 2 \chi_{0} \cos \tilde{\xi}\right)$.

To obtain a bound on the coefficients $\left(k_{F}\right)_{\kappa \lambda \mu \nu}$, our strategy is to fit Eq. (9) to polarization measurements of distant sources at multiple wavelengths. Since a single source constrains only $\sigma$ and $\tilde{\xi}$ in the particular primed frame appropriate for that one source, a measurement involves only a two-dimensional surface in the ten-dimensional coefficient space. It follows that at least five different sources are required to make a complete measurement of all the coefficients $k^{a}$.

Since the sensitivity to $k^{a}$ is inversely related to wavelength, polarimetry at shorter wavelengths yields better bounds. To maximize the sensitivity, we restrict attention to a chosen sample of 16 sources with published polarimetric data well suited to our purpose. Table I lists this sample, which spans wavelengths in the range $400-2200 \mathrm{~nm}$. For each source, we choose $\psi_{0}$ as the mean polarization angle and use Eq. (9) to create a $\chi^{2}$ distribution for $\delta \psi$ as a function of $\sigma, \tilde{\xi}, \lambda_{0}, \chi_{0}$. For each $\sigma, \tilde{\xi}$, we minimize with respect to $\lambda_{0}$ and $\chi_{0}$, which produces values consistent with expectations.

Figure 1 shows the change in the minimized distributions from their least value for the source 3CR 68.1. The

TABLE I. Source data.

\begin{tabular}{lcccc}
\hline \hline \multicolumn{1}{c}{ Source } & $L(\mathrm{Gpc})$ & $10^{30} L / \lambda$ & $\log _{10} \sigma$ & Ref. \\
\hline IC 5063 & 0.04 & $0.56-2.8$ & -30.8 & {$[6]$} \\
3A 0557-383 & 0.12 & $2.2-8.4$ & -31.2 & {$[7]$} \\
IRAS 18 325-5925 & 0.07 & $1.0-4.9$ & -31.0 & {$[7]$} \\
IRAS 19580-1818 & 0.13 & $1.8-9.1$ & -31.0 & {$[7]$} \\
3C 324 & 1.69 & $58-130$ & -32.2 & {$[8]$} \\
3C 256 & 1.92 & $70-140$ & -32.2 & {$[9]$} \\
3C 356 & 1.62 & $57-120$ & -32.2 & {$[10]$} \\
F J084044.5+ 363 328 & 1.71 & $62-120$ & -32.2 & {$[11]$} \\
F J155633.8+ 351 758 & 1.82 & $67-110$ & -32.2 & {$[11]$} \\
3CR 68.1 & 1.70 & $59-130$ & -32.2 & {$[12]$} \\
QSO J2359-1241 & 1.48 & $87-90$ & -31.1 & {$[13]$} \\
3C 234 & 0.55 & $51-75$ & -31.7 & {$[14]$} \\
4C 40.36 & 2.02 & $73-160$ & -32.2 & {$[15]$} \\
4C 48.48 & 2.04 & $75-160$ & -32.2 & {$[15]$} \\
IAU 0211-122 & 2.04 & $74-160$ & -32.2 & {$[15]$} \\
IAU 0828 + 193 & 2.08 & $78-160$ & -32.2 & {$[15]$} \\
\hline \hline
\end{tabular}

contour with $\Delta \chi^{2} \equiv \chi^{2}-\chi_{\text {min }}^{2}=50$ is displayed in the $\tilde{\xi}-\log \sigma$ plane. This corresponds to confidence level $\left(100-10^{-9}\right) \%$, which we regard as sufficient for a definite bound. Only $-90^{\circ} \leq \tilde{\xi} \leq 90^{\circ}$ need be considered, since $\delta \psi$ is symmetric under $\tilde{\xi} \rightarrow \tilde{\xi} \pm 180^{\circ}$. The general shape of this plot is common to all sources. Each source eliminates some region of coefficient space away from $\sigma=0, \tilde{\xi}=0, \pm 90^{\circ}$, which are the only configurations for which the theory predicts no change in the polarization angle. In principle, by combining data from multiple sources it would be possible to eliminate the regions near $\tilde{\xi}=0, \pm 90^{\circ}$ for all the sources. However, in the present context it suffices to make the reasonable assumption that the true signal does not lie in these regions for all the sources. Under this assumption, we have chosen conservative bounds on $\sigma$ for each source, listed in the fourth column of Table I. For the source 3CR 68.1, the bound is shown as a horizontal line across Fig. 1.

To estimate the constraint on $k^{a}$ that results from combining these bounds, we assume that the data for each source are consistent with $\sigma=0$. The bounds on $\sigma$ can then be regarded as conservative estimates of the error $\delta \sigma$ in a null measurement of $\sigma$. We then create a second $\chi^{2}$ distribution, $\chi^{2}=\sum_{j}\left(\sigma_{j}\right)^{2} /\left(\delta \sigma_{j}\right)^{2}$, where $j$ ranges over the 16 sources. Constant values of this distribution correspond to ten-dimensional ellipsoids in the coefficient space. Taking the magnitude $\left|k^{a}\right|=\sqrt{k^{a} k^{a}}$ of $k^{a}$ as the variable of interest and minimizing $\chi^{2}$ with respect to the other 9 degrees of freedom produces a constraint on $\left|k^{a}\right|$. Our conservative estimates yield $\left|k^{a}\right|<3 \times 10^{-32}$ at the 90\% confidence level.

As a check on this constraint, we have performed a grid search on the ten-dimensional space of coefficients $k^{a}$. Fixing the magnitude of the ten-dimensional vector $k^{a}$ in Eq. (7) leaves a nine-dimensional sphere. For a given point on the sphere, $\sigma$ and $\tilde{\xi}$ for each source can be found and the value of $\chi^{2}$ obtained using the minimized $\chi^{2}$ plots. Summing over sources yields a net $\chi^{2}$ minimized with respect to all parameters other than the coefficients $k^{a}$. A grid search on the nine-sphere with points separated by about 5 degrees of arc was used to find the minimum net $\chi^{2}$. Repeating for different values of $\left|k^{a}\right|$ gives results confirming our bound. To check our procedure directly, we also generated synthetic polarization data with a known

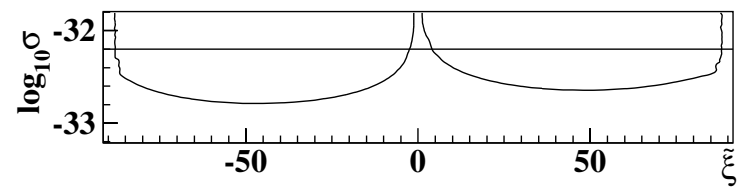

FIG. 1. Contour with $\Delta \chi^{2}=50$ for the source 3CR 68.1. 
faux Lorentz-violating signal and verified that our procedure correctly extracts it. Note that the absence of a signal emerging from our analysis indicates that systematic effects are irrelevant. To generate a false signal, these would need to mimic the expected direction and wavelength dependence.

No previous bounds on the coefficients $\left(k_{F}\right)_{\kappa \lambda \mu \nu}$ exist. However, constraints on different Lorentz-violating coefficients in the fermion sector of the standard-model extension have been obtained from studies of neutralmeson oscillations [18-21], comparative tests in Penning traps [22-25], spectroscopy of hydrogen and antihydrogen [26,27], measurements of muon properties [28,29], clock-comparison experiments [30-32], observations of the behavior of a spin-polarized torsion pendulum $[33,34]$, and studies of the baryon asymmetry [35]. The constraint reported here is comparable to the best of these existing limits, presently a few parts in $10^{31}$.

An improved bound could be obtained as more highquality data become available, particularly if measurements of the polarization angle $\chi$ could be incorporated in the analysis. Also, the sensitivity to inverse wavelength implies that spectropolarimetry of cosmological sources at frequencies above the ultraviolet band would be of interest. The technology to undertake $\mathrm{x}$-ray polarimetry of cosmological sources is presently being developed [36], which suggests an improvement of several orders of magnitude may eventually be attainable.

This work was supported in part by DOE Grant No. DEFG02-91ER40661 and by NASA Grant No. NAG8-1770.

[1] For overviews of various theoretical ideas, see, for example, CPT and Lorentz Symmetry, edited by V. A. Kostelecký (World Scientific, Singapore, 1999).

[2] V. A. Kostelecký and S. Samuel, Phys. Rev. D 39, 683 (1989); 40, 1886 (1989); Phys. Rev. Lett. 63, 224 (1989); 66, 1811 (1991); V. A. Kostelecký and R. Potting, Nucl. Phys. B359, 545 (1991); Phys. Lett. B 381, 89 (1996); Phys. Rev. D 63, 046007 (2001); V. A. Kostelecký, M. Perry, and R. Potting, Phys. Rev. Lett. 84, 4541 (2000).

[3] S. M. Carroll et al., Phys. Rev. Lett. 87, 141601 (2001); Z. Guralnik et al., Phys. Lett. B 517, 450 (2001); A. Anisimov et al., hep-ph/0106356.

[4] D. Colladay and V. A. Kostelecký, Phys. Rev. D 55, 6760 (1997); 58, 116002 (1998); Phys. Lett. B 511, 209 (2001); V. A. Kostelecký and R. Lehnert, Phys. Rev. D 63, 065008 (2001).

[5] We disregard here a possible $C P T$-odd Lorentz-violating term in the standard-model extension of the form $\frac{1}{2}\left(k_{A F}\right)^{\kappa} \epsilon_{\kappa \lambda \mu \nu} A^{\lambda} F^{\mu \nu}$ because it is expected to vanish for theoretical reasons [4] and is constrained experimentally by cosmological birefringence to levels well below those relevant here. See S.M. Carroll, G. B. Field, and R. Jackiw, Phys. Rev. D 41, 1231 (1990); R. Jackiw and V. A. Kostelecký, Phys. Rev. Lett. 82, 3572 (1999); J. F. C.
Wardle, R. A. Perley, and M.H. Cohen, Phys. Rev. Lett. 79, 1801 (1997), and references therein.

[6] J. H. Hough et al., Mon. Not. R. Astron. Soc. 224, 1013 (1987).

[7] C. Brindle et al., Mon. Not. R. Astron. Soc. 244, 577 (1990).

[8] A. Cimatti et al., Astrophys. J. 465, 145 (1996).

[9] A. Dey et al., Astrophys. J. 465, 157 (1996).

[10] A. Cimatti et al., Astrophys. J. 476, 677 (1997).

[11] M. S. Brotherton et al., Astrophys. J. 487, L113 (1997).

[12] M. S. Brotherton et al., Astrophys. J. 501, 110 (1998).

[13] M. S. Brotherton et al., Astrophys. J. 546, 134 (2001).

[14] M. Kishimoto et al., Astrophys. J. 547, 667 (2001).

[15] J. Vernet et al., Astron. Astrophys. 366, 7 (2001).

[16] M. Born and E. Wolf, Principles of Optics (Pergamon, New York, 1975), 5th ed.; J. Tinbergen, Astronomical Polarimetry (Cambridge University Press, New York, 1996).

[17] For a source at declination $d$ and right ascension $r$, the direction of propagation towards the Earth is $\hat{p}^{\mu}=$ $(1 ;-\cos d \cos r,-\cos d \sin r,-\sin d)$. The matrix

$$
R^{j k}=\left(\begin{array}{ccc}
\sin d \cos r & \sin d \sin r & -\cos d \\
\sin r & -\cos r & 0 \\
-\cos d \cos r & -\cos d \sin r & -\sin d
\end{array}\right)
$$

specifies the rotation between the celestial equatorial and primed frames.

[18] V. A. Kostelecký, Phys. Rev. Lett. 80, 1818 (1998); Phys. Rev. D 61, 016002 (2000); 64, 076001 (2001).

[19] KTeV Collaboration, Y. B. Hsiung et al., Nucl. Phys. Proc. Suppl. 86, 312 (2000).

[20] V. A. Kostelecký and R. Potting, Phys. Rev. D 51, 3923 (1995); D. Colladay and V. A. Kostelecký, Phys. Lett. B 344, 259 (1995); Phys. Rev. D 52, 6224 (1995); V. A. Kostelecký and R. Van Kooten, Phys. Rev. D 54, 5585 (1996); N. Isgur et al., Phys. Lett. B 515, 333 (2001).

[21] OPAL Collaboration, R. Ackerstaff et al., Z. Phys. C 76, 401 (1997); DELPHI Collaboration, M. Feindt et al., Report No. DELPHI 97-98 CONF 80, 1997; BELLE Collaboration, K. Abe et al., Phys. Rev. Lett. 86, 3228 (2001).

[22] R. Bluhm et al., Phys. Rev. Lett. 79, 1432 (1997); Phys. Rev. D 57, 3932 (1998).

[23] G. Gabrielse et al., Phys. Rev. Lett. 82, 3198 (1999).

[24] H. Dehmelt et al., Phys. Rev. Lett. 83, 4694 (1999).

[25] R. Mittleman et al., Phys. Rev. Lett. 83, 2116 (1999).

[26] R. Bluhm et al., Phys. Rev. Lett. 82, 2254 (1999).

[27] D. F. Phillips et al., Phys. Rev. D 63, 111101 (2001).

[28] R. Bluhm et al., Phys. Rev. Lett. 84, 1098 (2000).

[29] V. W. Hughes et al., Phys. Rev. Lett. 87, 111804 (2001).

[30] V. A. Kostelecký and C. D. Lane, Phys. Rev. D 60, 116010 (1999); J. Math. Phys. (N.Y.) 40, 6245 (1999).

[31] L. R. Hunter et al., in Ref. [1], and references therein.

[32] D. Bear et al., Phys. Rev. Lett. 85, 5038 (2000).

[33] R. Bluhm and V. A. Kostelecký, Phys. Rev. Lett. 84, 1381 (2000).

[34] B. Heckel et al., in Elementary Particles and Gravitation, edited by B. N. Kursunoglu et al. (Plenum, New York, 1999).

[35] O. Bertolami et al., Phys. Lett. B 395, 178 (1997).

[36] E. Costa et al., Nature (London) 411, 662 (2001). 